\title{
SIMPLE MODELS OF GALACTIC BULGES
}

\author{
N.W. EVANS \\ Institute of Astronomy \\ Madingley Rd \\ Cambridge \\ CB? 0 OHA
}

\begin{abstract}
We present a simple axisymmetric model with an elementary distribution function capable of representing galactic bulges. The gravity field of the galaxy is based on the axisymmetric logarithmic potential, which has a flat rotation curve. Bulges are built as isothermal distributions of stars embedded within the potential.
\end{abstract}

\section{Introduction}

Using cylindrical polar coordinates $(R, \phi, z)$, the axisymmetric logarithmic potential is (Binney, 1981)

$$
\psi=-\frac{1}{2} v_{0}^{2} \log \left(R_{c}^{2}+R^{2}+\frac{\tilde{z}^{2}}{q^{2}}\right),
$$

where $R_{c}$ is the core radius and $q$ is the axis ratio of the spheroidal equipotentials. We shall exploit the simplicity of the axisymmetric logarithmic potential to build bulge models. Suppose the bulge density embedded within the galaxy with gravity field (1) is

$$
\rho_{\text {bulge }}=\frac{\rho_{0} R_{c}^{p}}{\left(R_{c}^{2}+R^{2}+z^{2} q^{-2}\right)^{p / 2}},
$$

where $\rho_{0}$ is the central density and $p \geq 2$ prescribes the asymptotic density decay. The strength of our model is that the (even part of the) bulge distribution function is just an isothermal, namely

$$
F_{\text {bulge }}=D \exp \left(p E / v_{0}^{2}\right)
$$

where $D$ is a constant

$$
D=\rho_{0} R_{c}^{p}\left(\frac{p}{2 \pi v_{0}^{2}}\right)^{3 / 2}
$$




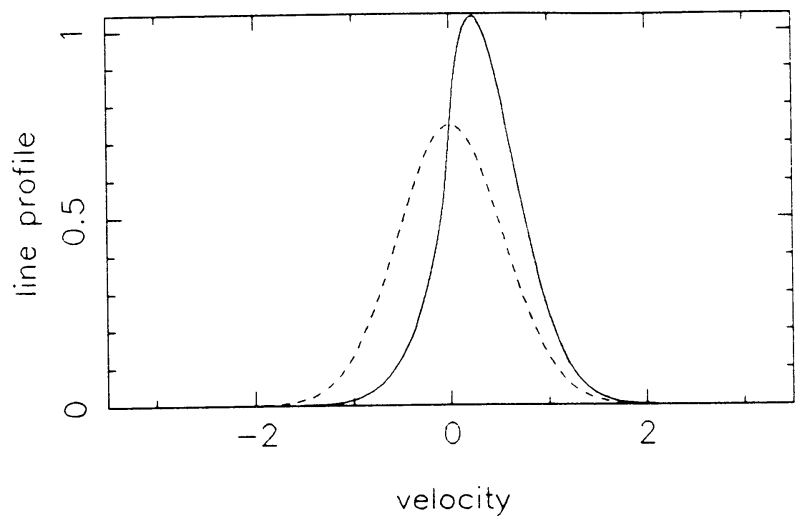

Fig. 1. Line profiles for rotating bulges

As an illustrative example, we shall take $p=3.1$ as the asymptotic density fall-off. Fixing the effective radius of the bulge as $2.0 \mathrm{kpc}$ gives $R_{c} \sim 1.9 \mathrm{pc}$. The circular velocity of the halo $v_{0}$ is taken as $\sim 220 \mathrm{kms}^{-1}$ and the axis ratio $q$ as 0.8 , while the central bulge density $\rho_{0} \sim 10^{5} M_{\odot} \mathrm{pc}^{-3}$. This gives an E2 bulge of total mass $\sim \tau \times 10^{\top} M_{0}$ embedded within the rest of the galaxy.

\section{Kinematic Properties}

All the properties of the bulge can be calculated very simply. For example, the velocity dispersion tensor is isotropic with all the second moments everywhere equal to the same value, i.e..

$$
\sigma_{R}^{2}=\sigma_{z}^{2}=\sigma_{\dot{\phi}}^{2}=\frac{v_{0}^{2}}{p} .
$$

With the parameters we have chosen, the velocity dispersion of bulge stars is $\sim 125 \mathrm{kms}^{-1}$. There is valuable kinematic information in the line profiles - that is, the distribution of line of sight velocities - derived from stellar absorption spectra. Generally, the line profile is very hard to calculate for models of bulges, but here the simplicity of our distribution function can be marvellously exploited. Without rotation, the line profile of our bulge is always exactly Gaussian, as shown in the dotted line in figure 1. Significant distortions from Gaussian only occur for rotating bulges - for example, the bold line is the line profile for the maximum streaming model. Further details are given in Evans (1992).

\section{References}

Binney, J.J., 1986 M. N.R. A. S., 196,

Eva.Ns, N.W., $1992 M . N . R . A . S$. in press. 